\title{
European Portuguese Accent in Acoustic Models for Non-native English Speakers
}

\author{
Carla Simões ${ }^{1,2}$, Carlos Teixeira ${ }^{2}$, Miguel Dias ${ }^{1}$, Daniela Braga ${ }^{1}$, \\ and António Calado ${ }^{1}$ \\ ${ }^{1}$ Microsoft Language Development Center, Av. Prof. Doutor Aníbal Cavaco Silva, \\ 2744-010 Porto Salvo, Portugal \\ ${ }^{2}$ Departamento de Informática, Faculdade de Ciências da Universidade de Lisboa, Campo \\ Grande, 1749-016 Lisboa, Portugal \\ \{t-carlas, midias, i-dbraga, i-antonc\}@microsoft.com, \\ carlos.teixeira@di.fc.ul.pt
}

\begin{abstract}
The development of automatic speech recognition systems poses several known difficulties. One of them concerns the recognizer's accuracy when dealing with non-native speakers of a given language. Normally a recognizer precision is lower for non-native users, hence our goal is to improve this low accuracy rate when the speech recognition system is confronted with a foreign accent. A typical usage scenario is to apply these models in applications where European Portuguese is dominant, but where English may also frequently occur. Therefore, several experiments were performed using cross-word triphone based models, which were then trained with speech corpora containing European Portuguese native speakers, English native speakers and English spoken by European Portuguese native speakers.
\end{abstract}

Keywords: Acoustic Models, European Portuguese accent, Speech Recognition.

\section{Introduction}

The tremendous growth of technology increased the need of integration of spoken language technologies into our daily applications, providing an easy and natural access to information. These applications are of different nature with different user interfaces. Besides voice enabled Internet portals or tourist information systems, Automatic Speech Recognition (ASR) systems can be used in home user experiences where TV and other appliances could be voice controlled, discarding keyboards or mouse interfaces, or in mobile phones and palm-sized computers for a hands-free and eyes-free manipulation.

When we think of the potential of ASR systems we must deal with the problem of language-dependency. This includes the non-native speaker's speech with different phonetic pronunciations from those of the language native speakers. The non-native accent can be more problematic than a dialect variation on the language. This mismatch depends on the individual speaking proficiency and speaker's 
mother tongue. Consequently, when the speaker's native language is not the same as the one that was used to train the recognizer, there is a considerable loss in recognition performance. Teixeira et al. [1], [2] have identified a drop of approximately $15 \%$ in the recognition accuracy when using a recognizer of native speakers. This paper describes several experiments and results using Portuguese speaker's using English, in order to improve the recognition performance of English language in a speech recognizer system within a European Portuguese dialogue system.

\subsection{Approach and Related Work}

A considerable number of methods and experiments for the treatment of non-native speech recognition has already been proposed by other authors. The simplest idea is to use non-native speakers' speech from a target language and train new acoustic models. This method is not reasonable because it can be very expensive to collect data that comprehends all the speech variability involved. An alternative is to apply speaker adaptation techniques such as Maximum Likelihood Linear Regression (MLLR) or Maximum A Posteriori (MAP) adaptation on speaker-independent context to reduce the disparity between the foreign accent and the native accent [3][12]. There is also research on the use of multilingual models using statistical data-driven models known as Hidden Markov Models (HMMs). The purpose is to develop standard acoustic models of phonemes where the similarities of sounds between languages are explored [4], [5], [6]. Another possibility is to include pronunciation variants to the lexicon of the recognizer using acoustic model interpolation [7], where each model of a native-speech recognizer is interpolated with the same model from a second recognizer which depends on the speaker's accent. Stefan Steidl et al. [8] considers that acoustic models of native speech are sufficient to adapt the speech recognizer to the way how non-native speakers pronounce the sounds of the target language. The HMM states from the native acoustic models are interpolated with each other in order to approximate the non-native pronunciation. Another approach is the training of selective data [11], where training samples from different sources are selected concerning a desired target task and acoustic conditions. The data is weighted by a confidence measure in order to control the influence of outliers. An appliance of such method is selecting utterances from a data pool which are acoustically close to the development data.

In this work we apply a number of acoustic modeling techniques to compare their performance on non-native speech recognition. The case of study is focused on English language spoken by European Portuguese speakers. Initially we explore the behavior of an English native model when tested with non-native speakers as well as the performance of a model only trained with non-native speakers. HMMs can be improved by retraining on suitable additional data. Regarding this we have trained a recognizer with a pool of accents, using utterances from English native speakers and English spoken by Portuguese speakers. Furthermore, we used adaptation techniques such as MLLR to reduce the variance between an English native model and the adaptation data, which in this case refers to the European Portuguese accent when speaking English language. To fulfill that task a native English speech recognizer is adapted using the non-native training data. Afterwards, we explore the pronunciation 
adaptation through adequate correspondences between phone sets of the foreign and target languages. Considering Bartkova et al. [6], we extended this approach when training new models with a new phone set, created by mapping the English and the Portuguese phone sets in order to support English words in a Portuguese dialogue system.

\subsection{Overview}

This article is organized as follows: the training and test corpora are presented in section 2. The training process, parameters and baseline systems are described in section 3. The several accomplished experiments such as pooling models, accent adaptation and new phone set mapping are described and discussed in section 4 . A brief conclusion and reference to future work is presented in section 5 .

\section{Corpora}

Our study was based on an experimental corpus of English spoken by European Portuguese speakers. This corpus is part of a larger one used in the Teixeira and Trancoso [2]. There are approximately 20 speakers (10 male and 10 female) for each accent, but only the male sub-set corpus was used in these experiments. To accomplish the experiments related with the application of the adaptation techniques or updating models, a native English Corpus (Teixeira and Trancoso) was used [2]. The audio files were sampled at $8 \mathrm{kHz}$ with 16 bits-linear precision. Each speaker has recorded two times approximately 227 English isolated words. The training and the test set are then separated to build a combined model for a speaker-independent speech recognition system. Table 1 shows the implied corpus and the partition for training and testing data set in this study.

Once the wave files were organized, the word level transcriptions were mapped into monophone level and then to triphone level. This procedure is further explained in the following sections. The phone sets of the languages presented in this study are defined using the SAMPA phonetic alphabet.

Table 1. Database overview

\begin{tabular}{ccccc}
\hline Data & Partition & Speakers & Utterances & Minutes \\
\hline Non-native & Training & 8 & 3468 & 35 \\
Data & Testing & 3 & 1221 & 12 \\
& Training & 7 & 3476 & 34 \\
\multirow{2}{*}{ Native Data } & Testing & 2 & 996 & 9 \\
\hline
\end{tabular}

\section{Acoustic Model}

All the experiments described in this paper are based on HMMs. Recognition was done using the Viterbi algorithm [9], used for obtaining the best sequence of states that match the sequences of speech frames that correspond to a certain unit. Our 
system is a cross-word triphone system and it is developed in an HTK based training tool [14] [15]. Each HMM has 3 states; each state represents a short segment of speech. This segment is the result of splitting the spoken utterances into frames of 10 milliseconds each. This representation is described mathematically by Gaussians probability distributions. Multiple mixture Gaussians are used and acoustically similar information is shared across HMMs by sharing/tying states called senones [16].

To train a set of HMMs, every file of training data must have an associated phone level transcription. The starting point of phone transcription is an orthographic transcription in HTK label format, a Master Label File (MLF), which is a single file containing a complete set of transcriptions. This allows the same transcriptions to be used with diferent versions of the speech data to be stored in diferent locations.

The training begins by converting word level transcriptions into monophone level transcriptions. Once reasonable monophone HMMs have been created, a forced alignment of the training data can be performed. Concerning this, a new phone level MLF is created in which the choice of pronunciations depends on the acoustic evidence. This new MLF can be used to perform a final re-estimation of the monophone HMMs. These models are iteratively updated by repeatedly traversing the training data and mapping the models to the monophone labels in the transcription.

After producing an initial monophones' model, the respective cross-word triphones are cloned for each monophone. This is done in two steps, first, the monophone transcriptions are converted to cross-word transcriptions and the cross-word triphones re-estimated to produce initial single-mixture models. Then, similar acoustic states of these triphones are clustered and tied to ensure that all state distributions can be robustly estimated. Since the system size is vastly reduced at this stage, we can increase the number of mixtures per senone. This leads to an initialized cross-word acoustic model, which is used to run through the training data and re-label the transcriptions to allow multiple pronunciations. After that these cross-word transcriptions are re-used to update the cross-word acoustic model leading once again to the final cross-word triphone system (Fig. 1 represents the training process described above) [14].

To carry out the experiments we have defined 1500 tying states (senones). For an initial number of mixtures we have a total of 12 mixtures and as a final smoothing stage we reduce the average total mixtures of the final system to 8 mixtures. For

\begin{tabular}{|l|c|c|}
\hline \multicolumn{4}{|c|}{ Feature Extraction } \\
\hline \multicolumn{4}{|c|}{ Monophone Generation } \\
\hline \multicolumn{4}{|c|}{ Alignment } & Update & Triphone Cloning \\
\hline Initialization & Triphone Clustering \\
\hline \multicolumn{4}{|c|}{ Alignment } & Mix-Down \\
\hline \multicolumn{4}{|c|}{ Triphone Training Model } \\
\hline \multicolumn{4}{|c|}{ Mixtering } & Mix-Up & Pruning & Final Training \\
\hline
\end{tabular}

Fig. 1. Training Acoustic Models Flowchart 
testing the several speech recognition systems we have defined a set of data dedicated to testing (see Table 1). Because we are talking about command and control systems, a grammar, which specifies the sequences of recognizable words, had to be defined. For that purpose a context free-grammar (CFG) was built with all the words found in the test set.

\subsection{Baseline Systems}

Both non-native and native acoustic models were trained with the respective training set described in Table 1. The training lexicon that lists the phonetic pronunciation of all the words in the corpus uses the English phone set. The parameters and training procedure are the same for the two models. The non-native and native speech engines were tested with the same corpus. Table 2 shows the performance on the non-native and native test set when using the both models. The remaining scores show better recognition performance when non-native models are used for the foreign accent.

Table 2. Accuracy rate on non-native and native data (WER \%)

\begin{tabular}{ccc}
\hline Models & $\begin{array}{c}\text { Non-Native } \\
\text { Models }\end{array}$ & $\begin{array}{c}\text { Native } \\
\text { Models }\end{array}$ \\
\hline Non-Native test set & $6.28 \%$ & $13.41 \%$ \\
Native test set & $22.89 \%$ & $4.09 \%$ \\
\hline
\end{tabular}

\section{Experiments and Results}

We explore different acoustic modeling methods to test their efficiency for recognition improvement on non-native speech. The results refer to experiments with cross-word triphone models which were obtained in a process reported in [14]. The different experiments and their results are described in the following sections.

\subsection{Pooled Models}

Non-native speech recognition can be viewed as speaker independent recognition problem, for which the traditional approach has been pooling all the speech data from as many speakers as possible as if it would belong to a single speaker. Pursuing this idea the native model was retrained with the available non-native data (pooled models). As we can see (Fig. 2) the improvement from pooling the native and nonnative training data indicates that recognition of non-native data can profit from native data. Since both corpus have almost the same training utterances, one way of weighting the non-native training utterances is to set up the weight parameter of the non-native training corpus.

The optimal weighting factor was found to be 2.0 for non-native data where recognition scores reveal a Word Error Rate (WER) of 6.02\% (non-native test) and $4.17 \%$ (native test). The recognition performance is slightly better when comparing these results with the English baseline system (Table 2). In [13] a pooled model using English native data and German accent shows an accuracy increasing of $1.2 \%$. 


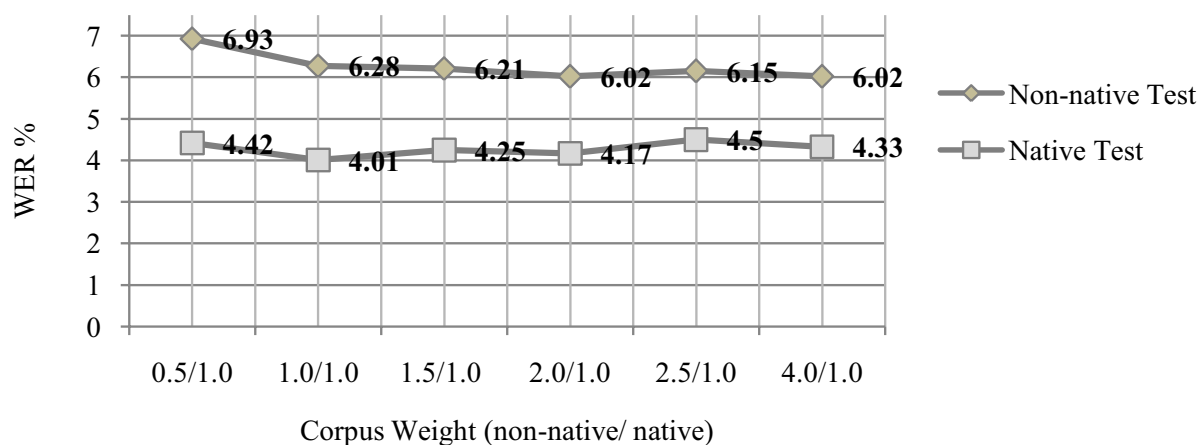

Fig. 2. Pooled models using different corpus weights for non-native corpus

\subsection{Adapting English Native Model}

The adaptation of acoustic models reduces the mismatch between a certain model set and the adaptation data. Adaptation can be at speaker level, environment or characteristics of a group of speakers such as foreign accent. The most used techniques are the Maximum Likelihood Linear Regression (MLLR) [12] and the maximum a-posteriori (MAP) [3]. MLLR computes a set of transformations, where one single transformation is applied to all models in a transformation class. More specifically it estimates a set of linear transformations for the context and variance parameters of a Gaussian mixture HMM system. The effect of these transformations is to shift the component means and to alter the variances in the initial system so that each state in the HMM system is more likely to generate the adaptation data.

In MAP adaptation we need a prior knowledge of the model parameter distribution. The model's parameters are re-estimated individually requiring more adaptation data to be effective. When larger amounts of adaptation training data become available, MAP begins to perform better than MLLR, due to this detailed update of each component.

Since we have a small amount of data in this experiment we used only the MLLR method, applied to English native model (baseline model) adapted with non-native corpus. Each model adaptation was iterated 4 times. Adaptation has improved performance on non-native recognition, revealing $6.21 \%$ WER for non-native test. On a native English test no changes were found, giving the same WER as the native model, $13.41 \%$.

\subsection{Mapping English Phonemes into Portuguese Phonemes}

Speaking a foreign language can change the native phonological structure (e.g. the English pharyngeal voiceless fricative in $<\underline{\text { hit }}>$ is commonly not articulated by Portuguese native speakers when speaking in English, since this phoneme is not present in the Portuguese phone set) or adapt unfamiliar sounds to similar/closer ones from their native phoneme inventory [6][10]. This also depends on the speaker proficiency, which will determine how different a native accent is from a foreign accent.

In order to get better recognition results on non-native accents, English phonemes were mapped into Portuguese phonemes. The mapping was done by a linguist expert who defined which phoneme inventory should be taken into account to describe a 
standard Portuguese English pronunciation. This phoneme inventory was selected bearing in mind the pronunciation of a Portuguese prestigious group/community, with a good knowledge of English language. As prestigious group we mean the higher literacy level group that uses a dominant variant or pattern dialect of a given language [17] [18].

Using the phonetic inventory SAMPA, 33 phones were defined to transcribe the English language when spoken by native Portuguese speakers. The majority of English phones suffered a direct mapping, except those that represent non Portuguese existing sounds. One example of that is the dental voiceless fricative [T] (e.g. $<$ thriller $>,<$ thirties $>$ ), that in European Portuguese language is converted to a dental voiceless plosive $[\mathrm{t}$ ] or alveolar voiceless fricative [s]. We consider that the prestigious group recognizes this sound so we have included it in our new phone set. Another non existing sound in European Portuguese is the pharyngeal voiceless fricative [h] (e.g. <hang >). However, for the prestigious group there is, for example, differentiation in pronunciation between $<$ and $>$ and $<$ hand $>$, which made us include this phone in the English-Portuguese mapped phone set.

Finally, another important difference we need to consider is the approximant $[\mathrm{r}]$ (e.g. $<$ red $>$ ). This phone does not exist in the European Portuguese phone set. Despite this, in some words, when a Portuguese speaker uses English, like $<\underline{\text { red }}>$, we can say [R E d] (PT SAMPA), in other cases such as <car> we say [k a r] (PT SAMPA). For this reason we added this phone to the new mapped phone set.

Afterwards, new word transcriptions of the corpus vocabulary were required, following an accurate process accomplished by the linguist expert. The initial English phonetic transcription was directly mapped to the Portuguese form (using the new phone set), which is not enough for a reliable representation of English spoken with a Portuguese accent. An improved phonetic transcription was accomplished by modifying the phonetic transcription of each word, taking into account the Portuguese prestigious accent while using English. An example of that adaptation is the way an English or a Portuguese speaker will pronounce words that end with a plosive consonant such as [t] (e.g < art>). In English we should transcribe the word <art> like [Q r t] (PT SAMPA) but in Portuguese we say [Q r t @] (PT SAMPA).

Results reveal $7.26 \%$ of WER for the new trained models using the phone set described above. The recognition accuracy has decreased, when comparing it with the baseline non-native system or the pooled model experiment, but it is still far from the English native model when tested with a non-native test set. This is an encouragement for continuing exploring this subject.

Another experiment was training a pooled model using this new phone set, but instead of using the English native model, we experimented it with a Portuguese native model. Doing this we are representing a European Portuguese recognition system that also supports English words with a Portuguese accent. The Portuguese native model was trained with 87 hours of speech for a total of 553 speakers (266 male and 287 female). The audio files were sampled at $16 \mathrm{kHz}$ with 16 bits-linear precision. The results of such experiment were encouraging, as the system reveals $9.81 \%$ of WER when testing with the non-native test set. This value is still above the baseline English system results, which means that an accurate phonetic representation may improve recognition performance of non-native speakers. 


\section{Conclusions}

In this paper we have explored several ways of adapting automatic speech recognition systems to non-native speakers. The results presented in this paper show that a small amount of data can be successfully used for the improvement of non-native accent recognition. Even though some applied methods reveal worst performance results when compared to the only non-native trained models, there are considerably improvements on the English native models recognizing non-native accents.

The following graphic (Fig. 3) gives the best results and its proportion between methods. As we can infer from the last experience, where we use the mapped phone set to train new models, the test using the English native test corpus was not performed. In this experiments we work with a different phone set from the one used in the English native model, so the results related with testing those models with English native speaking are not relevant for the experiment.

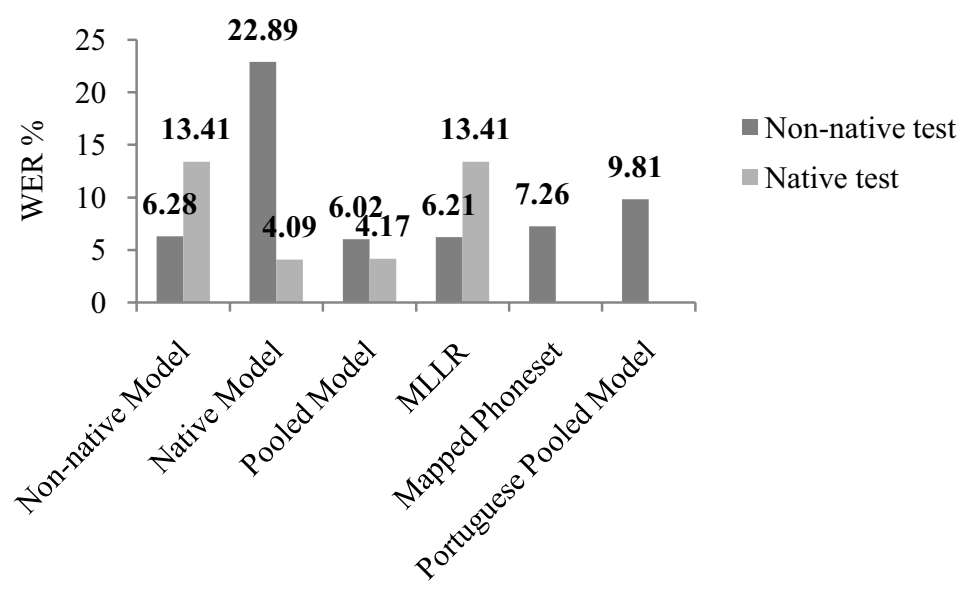

Fig. 3. Best results of the different experiments

In the future, we plan to explore some of these methods, especially to continue the study of pronunciation in the context of Portuguese speaking foreign words, in order to improve recognition performance of dialogue systems.

\section{References}

1. Teixeira, C., Trancoso, I., Serralheiro, A.: Recognition of Non-Native Accents. In: Eurospeech, vol. 5, pp. 2375-2378 (1997)

2. Teixeira, C., Trancoso, I.: Word Rejection using Multiple Sink Models. In: Proc. ICSLP, pp. 1443-1446, Banff (1992)

3. Zavaliagkos, G., Schwartz, R., Makhoul, I.: Batch, Incremental and Instantaneous Adaptation Techniques for Speech Recognition. In: Proc. ICASSP (1995)

4. Kunzmann, S., Fischer, V., Gonzalez, J., Emam, O., Gunther, C., Janke, E.: Multilingual Acoustic Models for Speech Recognition and Synthesis, IEEE. In: ICASSP (2004) 
5. Kohler, J.: Multi-lingual Phoneme Recognition Exploiting Acoustic-Phonetic Similarities of Sounds, Siemens AG, Munich, Germany

6. Bartkova, K., Jouvet, D.: Multiple models for improved speech recognition for non-native speakers. In: SPECOM (2004)

7. Livescu, K., Glass, J.: Lexical Modelling of Non-Native Speech for Automatic Speech Recognition. In: ICASSP (2000)

8. Steidl, S., Stemmer, G., Hacker, C., Nöth, E.: Adaptation in the Pronunciation Space for Non-Native Speech Recognition. In: ICSLP, Korea (2004)

9. Rabiner, L.R.: A tutorial on hidden Markov models and selected applications in speech recognition. Proceedings of the IEEE 77(2), 257-286 (1989)

10. Flege, J.E., Schirru, C., MacKay, I.: Interaction between the native and second language phonetic subsystems. Speech Communication 467-491 (2003)

11. Arslan, L.M., Hansen, J.H.L.: Selective Training in Hidden Markov Model Recognition. IEEE Transactions on Speech and Audio Processing 7(1), 46-54 (1999)

12. Leggetter, C.J., Woodland, P.C.: Speaker Adaptation of HMMs Using Linear Regression (1994)

13. Wung, Z., Schultz, T., Waibel, A.: Comparison of Acoustic Model Adaptation Techniques on Non-Native Speech, IEEE. In: ICASSP (2003)

14. Morton, R.: The Training Guide, A Guide for Training Acoustic Models, Microsoft Document

15. Young, S., Kershaw, D., Odell, J., Ollason, D., Valtchev, V., Woodland, P.: The HTK Book (1999)

16. Woodland, P., Young, S.: The HTK Tied-State Continuous Speech Recognition. In: Proc. Eurospeech (1993)

17. Ferreira: Variação linguística: perspectiva dialectológica, in Faria et al. 1996. Introdução á Linguística Geral e Portuguesa,Lisboa, Caminho: 483

18. Fromkin, Rodman: Introdução á Linguagem, Coimbra, Almedina: 273 